\title{
ДИНАМИКА ВЯЗКОИ НЕСЖИМАЕМОЙ ЖИДКОСТИ В ГИЛЬБЕРТОВОМ ПРОСТРАНСТВЕ С УЧЕТОМ КРАЕВЫХ УСловий
}

\author{
(Представил Я. Эйнасто)
}

\section{1. Постановка задачи}

Рассмотрим движение жидкости в двумерной области

$$
\Omega=\left\{0<x_{1}<a^{-1}, 0<x_{2}<1\right\}, \quad 0<a \leqslant 1,
$$

которое описывается уравнениями Навье-Стокса

$$
\begin{gathered}
\mathbf{y}_{, t}+r v^{\alpha} \mathbf{v}, \alpha-\Delta \mathbf{v}-\mathbf{f}=-\operatorname{grad} p, \quad \mathbf{x} \in \Omega, \\
\operatorname{div} \mathbf{v}=0, \quad \mathbf{x} \in \Omega,
\end{gathered}
$$

где $r \geqslant 0$ - число Рейнольдса, а $\mathbf{x}, t, \mathbf{v}=\mathbf{v}(\mathbf{x}, t), p=p(\mathbf{x}, t), \mathbf{f}=\mathbf{f}(\mathbf{x})$ безразмерные координаты, время, скорость, давление и заданное силовое поле. По повторяющимся греческим индексам происходит везде суммирование.

Относительно $\left.\mathbf{v}\right|_{t=0}$ предположим, что она удовлетворяет условию несжимаемости (3) и заданным краевым условиям. Будем рассматривать следующие два набора краевых условий.

Первая краевая задача:

$$
\begin{array}{ll}
\left.\mathbf{v}\right|_{x_{1}=0}=\left.\mathbf{v}\right|_{x_{1}=a^{-1}} \forall x_{2} & \text { (условие } a^{-1} \text {-периодичности), } \\
\left.\mathbf{v}\right|_{x_{2}=0}=0,\left.\mathbf{v}\right|_{x_{2}=1}=\mathbf{e}^{1} b \forall x_{1} & \text { (условия прилипания), }
\end{array}
$$

где $\mathrm{e}^{\alpha}-$ единичный вектор в направлении координатной оси $x_{\alpha}$, a $b-$ неотрицательная постоянная.

Вторая краевая задача:

$$
\left.\mathbf{v}\right|_{\Gamma}=\mathbf{b}(\mathbf{x}), \quad \oint_{\Gamma} \mathbf{b} d \mathbf{s}=0,
$$

где $\Gamma-$ контур области $\Omega$.

Условие $a^{-1}$-периодичности $\mathbf{v}$ накладывает в случае первой краевой задачи требование $a^{-1}$-периодичности на все члены уравнения $(2)$ :

$$
\mathbf{v},\left.t\right|_{x_{1}=0}=\mathbf{v},\left.t\right|_{x_{1}=a^{-1}}, \quad v^{\alpha} \mathbf{v},\left.\alpha\right|_{x_{1}=0}=v^{\alpha} \mathbf{v},\left.\alpha\right|_{x_{1}=a^{-1}} \text { и т. д. }
$$

Уравнение (2) можно заменить вариационным уравнением

$$
\int_{\Omega}\left[(\mathbf{v}, t-\mathbf{f}) \mathbf{w}-\left(r v^{\alpha} \mathbf{v}-\mathbf{v}_{, \alpha}\right) \mathbf{w}_{, \alpha}\right] d x=0 \quad \forall \mathbf{w} \in V, t,
$$

которое представляет собой основное уравнение механики для вязкой несжимаемости жидкости и выражает условие ее нулевой работы при произвольных виртуальных движениях w $\left[{ }^{1-3}\right]$. Строгое математическое содержание придается уравнению (6) в рамках теории обобщенных функций $\left[{ }^{4-6}\right]$. 
Пространство $V$, элементом которого является виртуальная скорость w., определяем следующим образом:

$$
\begin{gathered}
V^{\prime}=\left[\mathrm{V}_{H^{\prime}}=\right. \\
\text { ช в в пространстве } H^{1} \text {. }
\end{gathered}
$$

В этом определении $H^{1}=W_{2}{ }^{1}(\Omega)$ - пространство Соболева (определение и свойства $H^{1}$ можно найти в $\left.\left[{ }^{4-7}\right]\right)$. Линейное многообразие ช в определении (7) зависит от типа краевой задачи:

$$
\begin{aligned}
& \text { первая - } v=\left\{\mathbf{w}\left|\mathbf{w} \in C_{p e r}^{1}, \operatorname{div} \mathbf{w}=0, \mathbf{w}\right|_{x_{2}=0}=\left.\mathbf{w}\right|_{x_{2}=1}=0\right\} ; \\
& \text { вторая - } v=\left\{\mathbf{w}\left|\mathbf{w} \in C^{1}, \operatorname{div} \mathbf{w}=0, \mathbf{w}\right|_{\mathrm{r}}=0\right\} .
\end{aligned}
$$

Здесь через $C^{1}$ обозначено множество непрерывно дифференцируемых в замкнутой области $\bar{\Omega}=\Omega+\Gamma$ вектор-функций. Линейное многообразие $C_{p e r}^{1}$ является подмножеством $C^{1}$ и состоит из функций, периодических в $\bar{\Omega}:\left.\mathbf{v}\right|_{x_{1}=0}=\left.\mathbf{v}\right|_{x_{1}=a^{-1}},\left.\mathbf{v}\right|_{x_{2}=0}=\left.\mathbf{v}\right|_{x_{2}=1}$.

Цель настоящей работы - получение уравнений движения для вязкой несжимаемой жидкости в ортогональном базисе с сохранением краевых условий. Сформулируем основные предпосылки, необходимые для решения поставленной задачи.

Будем предполагать, что решение уравнения (6) можно представить в виде

$$
\mathbf{v}=\mathbf{u}+\mathbf{v}_{0},
$$

где $\mathbf{v}_{0}$ - заданное гладкое соленоидальное поле, удовлетворяющее краевым условиям (4) или (5). В качестве функции $\mathbf{v}_{0}$ можно выбрать решение соответствующей стационарной задачи, но это не обязательно. Новая динамическая переменная и удовлетворяет включению

$$
\mathbf{u} \in V \mathbf{v} t .
$$

Если ввести в рассмотрение функциональное пространство

$$
\mathfrak{H}=\left[C_{p e r}^{1}\right]_{H^{1}}
$$

то для обеих краевых задач будет иметь место вложение

$$
V \subset \mathcal{H} \text {. }
$$

Пространство $\mathscr{X}$ - сепарабельное гильбертово пространство с нормой, индуцированной из $H^{1}$. В пространстве $\mathcal{J}$ (а также в его комплексном расширении) образует базис система функций

$$
\begin{aligned}
& \vec{\psi}_{m n}^{\alpha}(x)=\mathrm{e}^{\alpha} \psi_{m n}(\mathbf{x}), \psi_{m n}(\mathbf{x})=\sqrt{a} \exp \left(i \mathbf{k}_{m n} \mathbf{x}\right), \\
& \mathbf{k}_{m n}=k_{m n}^{\alpha} \mathbf{e}^{\alpha}, k_{m n}^{1}=2 \pi a m, k_{m n}^{2}=2 \pi n .
\end{aligned}
$$

Вследствие вложения (12) решение $\mathbf{u}(\mathbf{x}, t)$ можно в каждый момент представить в виде двукратного ряда Фурье

$$
\mathbf{u}(\mathbf{x}, t)=\sum_{m, n} u_{m n}^{\alpha}(t) \vec{\psi}_{m n}^{\alpha}(\mathbf{x}),\|\mathbf{u}\|_{H}^{2}=\sum_{m, n} u_{m n}^{\alpha}\left(u_{m n}^{\alpha}\right)^{*}\left(1+\mathbf{k}_{m n}^{2}\right) .
$$

Здесь $\left(u_{m n}^{\alpha}\right)^{*}$ означает комплексно сопряженную с $u_{m n}^{\alpha}$ величину.

Обстоятельство, что действительная функция u разлагается в комплексном базисе, накладывает дополнительные условия на компоненты $u_{m n}^{\alpha}:\left(u_{m n}^{\alpha}\right)^{*}=u_{-m,-n}^{\alpha}$. Мы, однако, снимаем эти ограничения при 
выводе уравнений движения в ортогональном представлении и будем рассматривать и как комплексную функцию, считая все пространства комплексными. Условие вещественности и можно всегда ввести в получаемые уравнения, требуя выполнения равенства $\left.\mathbf{u}\right|_{t=0}=\left.\mathbf{u}^{*}\right|_{t=0}$. Предпочтение экспоненциальному базису (13) перед тригонометрическим базисом Фурье вызвано рядом причин, среди которых важнейшей является диагональность операторов дифференцирования в этом базисе.

Вывод уравнений движения в ортогональном представлении будет осуществлен в два этапа.

На первом этапе будем рассматривать конечномерные системы, предполагая, что при любом $t$ можно представить динамическую переменную и конечномерным рядом Фурье

$$
\mathbf{u}=\sum_{|m| \leqslant M} \sum_{n \mid \leqslant N} u_{m n}^{\alpha} \vec{\psi}_{m n}^{\alpha} .
$$

Если ввести в рассмотрение $2(2 M+1)(2 N+1)$-мерное пространство $L(M, N)$ комплексных последовательностей $\left\{u^{\alpha}{ }_{m n}, \alpha=1,2 ; m=\right.$ $=-M, \ldots, M ; n=-N, \ldots, N\}$ с нормой

$$
\|\mathbf{u}\|_{L(M, N)}^{2}=\sum_{|m| \leqslant M} \sum_{|n| \leqslant N} u_{m n}^{\alpha}\left(u_{m n}^{\alpha}\right)^{*},
$$

то $\mathbf{u}$ будет описываться изображающей точкой в этом пространстве, а динамика жидкости - аппроксимироваться динамикой конечной механической системы в $L(M, N)$.

На втором этапе будет осуществлен предельный переход $M, N \rightarrow \infty$ и будет показано, что при этом уравнения движения в ортогональном представлении приобретают точность в пространстве $V$.

Такой подход несколько упрощает изложение предмета. На первом этапе имеем дело с конечной механической системой, к которой примени́м принцип виртуальной работы. Таким образом, внимание сосредотачивается главным образом на механической природе движения. Проблемы же чисто математического характера, присущие бесконечным динамическим системам, возникают лишь на втором этапе. Кроме того, такой подход приводит к пространству $V$ как пределу конечномерных конструктивно определяемых пространств. И, наконег, конечные системы в $L(M, N)$ представляют собой самостоятельный интерес как математические модели, отражающие основные физические закономерности движения вязкой несжимаемой жидкости, стесненной краевыми условиями.

\section{2. Уравнения движения в пространстве $L(M, N)$}

Рассмотрим подробно вывод уравнения движения для первой краевой задачи (вывод других уравнений аналогичен). Центр внимания при этом падает на виртуальное пространство, которое является подпространством $L(M, N)$ и определяется кинематическими условиями, налагаемыми на вектор (14). Значение виртуального пространства состоит в том, что в конечном счете динамика проектируется в это пространство.

Вектор (14) должен приं любом $t$ удовлетворять условию соленоидальности

$$
\operatorname{div} \mathbf{u}=\sum_{m, n} u_{m n}^{\alpha} \operatorname{div} \vec{\psi}_{m n}^{\alpha}=i \sum_{m, n} u_{m n}^{\alpha} k_{m n}^{\alpha} \psi_{m n}=0
$$


и краевым условиям, которые для и в случае первой краевой задачи имеют вид

$$
\left.\mathbf{u}\right|_{x_{2}=0 ; 1}=\left.\sum_{m, n} u_{m n}^{\alpha} \vec{\psi}_{m n}^{\alpha}\right|_{x_{2}=0 ; 1}=\sqrt{a} \sum_{|m| \leqslant M} \exp \left(i 2 \pi a m x_{1}\right) \sum_{|n| \leqslant N} u_{m n}^{\alpha} \mathbf{e}^{\alpha}=0 .
$$

Из этих равенств получим с учетом линейной независимости $\psi_{m n}$, $\exp \left(i 2 \pi a m x_{1}\right)$ и ортогональности $\mathrm{e}^{\alpha}$ условия, которым должна удовлетворять изображающая точка $\left\{u^{\alpha}{ }_{m n}\right\}$ :

$$
u_{m n}^{\alpha} k_{m n}^{\alpha}=0, \quad|m| \leqslant M, \quad|n| \leqslant N
$$

(условия соленоидальности),

$$
\sum_{|n| \leqslant N} u_{m n}^{\alpha}=0, \quad \alpha=1,2, \quad|m| \leqslant M
$$

(краевые условия).

Система (15)-(16) определяет пространство (гиперплоскость) в $L(M, N)$, которое обозначим через $V(M, N)$. Вычислим размерность этого пространства. Система (15) состоит из $(2 M+1)(2 N+1)-1$ независимых уравнений (при $m=0, n=0$ имеем $k^{\alpha}{ }_{m n}=0$, и (15) удовлетворяется тождественно), а система (16) - из $2(2 M+1)$ независимых уравнений. Следовательно,

$$
\begin{gathered}
\operatorname{dim} V(M, N)=\operatorname{dim} L(M, N)-(2 M+1)(2 N+1)+1-2(2 M+1)= \\
=(2 M+1)(2 N-1)+1 .
\end{gathered}
$$

Уравнения (15) и (16) будем рассматривать как связи, обусловливающие появление сил реакции в динамической системе. Таким образом, динамика жидкости в $L(M, N)$ - это динамика стесненной механической системы, а $V(M, N)$ - пространство виртуальных скоростей. Уравнения движения стесненной связями системы легче всего получить с помощью вариационного уравнения.

Согласно основным положениям механики $\left[{ }^{3}\right]$, виртуальные скорости $\mathbf{w}$ принадлежат пространству $V(M, N)$. Из однородности связей (15) и (16) следует, что они, будучи представлены как элементы пространства $L(M, N)$, удовлетворяют тем же ограничениям, что и $\mathbf{u}$

$$
w_{m n}^{\alpha} k_{m n}^{\alpha}=0, \quad \sum_{|n| \leqslant N} w_{m n}^{\alpha}=0 .
$$

Подставим разложение

$$
\mathbf{w}=\sum_{|m| \leqslant M} \sum_{|n| \leqslant N} w_{m n}^{\alpha}\left(\vec{\psi}_{m n}^{\alpha}\right)^{*}
$$

(комплексное сопряжение взято для удобства) и (10) в уравнение (6). Получим

$$
\sum_{|m| \leqslant M} \sum_{|n| \leqslant N}\left[\dot{u}_{m n}^{\alpha}+i k_{m n}^{\beta} F_{m n}^{\beta \alpha}\left(\mathbf{u}+\mathbf{v}_{0}\right)-f_{m n}^{\alpha}\right] w_{m n}^{\alpha}=0
$$

где

$$
\begin{gathered}
\dot{u}_{m n}^{\alpha} \equiv d u_{m n}^{\alpha} / d t, \quad f_{m n}^{\alpha}=\left(\mathbf{f}, \vec{\psi}_{m n}^{\alpha}\right)=\int_{\Omega} \mathbf{f}\left(\vec{\psi}_{m n}^{\alpha}\right)^{*} d x, \\
F_{m n}^{\alpha \beta}(\mathbf{v})=\int_{\Omega}\left(r v^{\alpha} v^{\beta}-v_{, \alpha}^{\beta}\right)\left(\psi_{m n}\right)^{*} d x .
\end{gathered}
$$


Варьирование этого уравнения по w с учетом условий (17) приводит к искомым динамическим уравнениям

$$
\begin{gathered}
\dot{u}_{m n}^{\alpha}+i k_{m n}^{\beta} F_{m n}^{\beta \alpha}\left(\mathbf{u}+\mathbf{v}_{0}\right)-f_{m n}^{\alpha}=k_{m n}^{\alpha} p_{m n}+\lambda_{m}^{\alpha}, \\
\alpha=1,2, \quad|m| \leqslant M, \quad|n| \leqslant N .
\end{gathered}
$$

Уравнения (15), (16), (18) образуют замкнутую сйстему для определения неизвестных $u^{\alpha}{ }_{m n}, p_{m n}, \lambda^{\alpha}$. Слагаемые в правой части уравнений (18) представляют собой силы реакции, вызванные связями (15) и $(16) ;\left\{p_{m n}\right\}$ - давление.

В случае второй краевой задачи виртуальное пространство $V(M, N)$ определяется уравнениями

$$
\begin{gathered}
u_{m n}^{\alpha} k_{m n}^{\alpha}=0, \quad|m| \leqslant M, \quad|n| \leqslant N, \\
\sum_{|n| \leqslant N} u_{m n}^{\alpha}=\rho,|m| \leqslant M, \quad \sum_{|m| \leqslant M} u_{m n}^{\alpha}=0, \quad|n| \leqslant N, \alpha=1,2,
\end{gathered}
$$

откуда следует, что $\operatorname{dim} V(M, N)=(2 M-1)(2 N-1)-3$. Уравнения движения имеют вид

$$
\dot{u}_{m n}^{\alpha}+i k_{m n}^{\beta} F_{m n}^{\beta \alpha}\left(\mathbf{u}+\mathbf{v}_{0}\right)-f_{m n}^{\alpha}=k_{m n}^{\alpha} p_{m n}+\lambda_{m}^{\alpha}+\gamma_{n}^{\alpha} .
$$

Эта система совместно с уравнениями связи (19) позволяет определить неизвестные $u^{\alpha}{ }_{m n}, p_{m n}, \lambda^{\alpha}{ }_{m}, \gamma^{\alpha}{ }_{n}$.

Полученные нами уравнения представляют собой первую форму уравнений движения в $(M, N)$-приближении. Ниже будут выведены эквивалентные уравнения, отличающиеся от записанных выбором пространства, элементом которого является и. Возможность описания движения системы в подпространствах пространства $L(M, N)$ подсказывается содержанием уравнений (18) и (20). Вектор u, хотя он и рассматривается как элемент $L(M, N)$, в действительности принадлсжит пространству $V(M, N)$. Удерживают вектор и на гиперповерхности $V(M, N)$ силы реакции (слагаемые правой части уравнений $(18),(20))$, которые, будучи ортогональными к поверхности $V(M, N)$, имеют в каждый момент такую величину, что $\dot{\mathrm{u}}=\left\{\dot{u}_{m n}^{\alpha}\right\}$ принадлежит $V(M, N)$. Вследствие этого вектор и не способен покинуть в своем движении пространство $V(M, N)$, если он находился там в начальный момент. Другими словами, силы реакции уравновешивают ортогональную к гиперповерхности $V(M, N)$ компоненту силы $\mathbf{F}=\left\{-i k_{m n}^{\beta} F^{\beta} \alpha_{m n}+f^{\alpha}{ }_{m n}\right\}$. Поэтому силы реакции можно исключить из уравнений движения, не изменив при этом динамики, что приведет к замене силы $\mathbf{F}$ eе ортогональной проекцией в пространство $V(M, N)$. Для этого есть два эквивалентных способа:

а) можно построить ортопроектор $\hat{P}: L(M, N) \rightarrow V(M, N)$, рассматривая и и $\mathbf{F}$ как элементы $L(M, N)$; уравнения движения приобретают при этом вид $\dot{\mathrm{u}}=\hat{P} \mathrm{~F}$;

б) можно ввести в пространство $V(M, N)$ базис и проектировать в него уравнения движения, принимая при этом вектор и за элемент пространства $V(M, N)$.

Ниже мы будем пользоваться в основном вторым способом.

\section{3. Динамика в пространстве соленоидальных векторов}

Перейдем к промежуточному пространству соленоидальных векторов $S(M, N)$, обладающему свойсттом 


$$
V(M, N) \subset S(M, N) \subset L(M, N) .
$$

Как следствие, получим в $S(M, N)$ уравнения, не содержащие давления. Пространства соленоидальных векторов используются в классической и квантовой электродинамике $\left[{ }^{8}\right]$, а также при моделировании гидродинамических полей $\left[{ }^{9}\right]$. Поэтому сформулируем здесь конечный результат. Отметим, однако, что к определению $S(M, N)$ как подпространству $L(M, N)$ можно прийти, исключая из полученных выше уравнений давление.

Заменим (13) новым базисом

где

$$
\vec{\varphi}_{m n}^{\alpha}(\mathbf{x})=\mathbf{e}_{m n}^{\alpha} \psi_{m n}(\mathbf{x})
$$

$$
\begin{gathered}
\mathbf{e}_{m n}^{\alpha}=\varrho_{m n}^{\alpha} \mathrm{e}^{1}+\sigma_{m n}^{\alpha} \mathrm{e}^{2}, \\
\mathrm{\varrho}_{00}^{1}=\sigma_{00}^{2}=1, \quad \mathrm{Q}_{00}^{2}=\sigma_{00}^{1}=0, \\
\mathrm{\varrho}_{m n}^{1}=\sigma_{m n}^{2}=k_{m n}^{2} /\left|\mathbf{k}_{m n}\right|, \quad \mathrm{Q}_{m n}^{2}=-\sigma_{m n}^{1}=k_{m n}^{1} /\left|\mathrm{k}_{m n}\right| .
\end{gathered}
$$

Легко проверить, что переход $\vec{\psi}_{m n}^{\alpha} \leftrightarrow \vec{\varphi}^{\beta}{ }_{m n}$ взаимно однозначен и что (21) является ортогональным базисом в пространстве $\mathscr{X}$.

Представим вектор (14) в базисе (21) следующим образом

$$
\mathbf{u}=\mathbf{u}_{\mathrm{s}}+\mathbf{u}_{G},
$$

где

$$
\begin{gathered}
\mathbf{u}_{S}={\overrightarrow{u^{0} \varphi_{00}^{2}}}^{\overrightarrow{2}_{|m|}} \sum_{\mid \leqslant M} \sum_{|n| \leqslant N} u_{m n} \vec{\varphi}_{m n}^{1}, \\
\mathbf{u}_{G}=\sum_{|m| \leqslant M} \sum_{|n| \leqslant N} \sum^{\prime} \tilde{u}_{m n} \vec{\varphi}_{m n}^{2},
\end{gathered}
$$

а штрих у двойной суммы означает, что здесь отсутствует слагаемое $m=n=0$. Можно убедиться, что $\operatorname{div} \vec{\varphi}_{00}^{2}=\operatorname{div} \vec{\varphi}_{m n}^{1}=0 \mathrm{Vm}, n ; \vec{\varphi}_{m n}^{2}=$ $=-i \operatorname{grad} \psi_{m n} /\left|\mathbf{k}_{m n}\right|, \operatorname{div} \vec{\varphi}_{m n}^{2}=i\left|\mathbf{k}_{m n}\right| \psi_{m n} \neq 0$, если $m$ или $n$ не равняется нулю. Поэтому $\mathbf{u}_{S}$ является всегда соленоидальным вектором, а $\mathbf{u}_{G}-$ градиентом. Отсюда заключаем, что $\mathbf{u}(\mathbf{x}, t)-$ решение вариационной задачй в $(M, N)$-приближении - представимо в базисе $\left\{\vec{\varphi}_{00}^{2}, \overrightarrow{\varphi_{m n}^{1}}\right\}$

$$
\mathbf{u}(\mathbf{x}, t)=u^{0}(t) \vec{\varphi}_{00}^{2}+\sum_{|m| \leqslant M} \sum_{|n| \leqslant N} u_{m n}(t) \vec{\varphi}_{m n}^{1}(x) .
$$

Всевозможные $(2 M+1)(2 N+1)+1$-мерные последовательности $\left\{u^{0}, u_{m n}\right\}$, наделенные метрикой, индуцированной из $L(M, N)$, и образуют пространство соленоидальных векторов $S(M, N)$.

Рассмотрим ограничения, налагаемые на вектор (23) в случае первой краевой задачи. Условие соленоидальности, очевидно, выполняется; краевые же условия дают

$$
\left.\mathbf{u}\right|_{x_{2}=0 ; 1}=\sqrt{a}\left[\sigma_{00}^{2} \mathrm{e}^{2} u^{0}+\sum_{|m|<M} \exp \left(i 2 \pi a m x_{1}\right) \sum_{|n|<N} \sum_{m n}\left(u_{m n} \mathbf{e}^{1}+\sigma_{m n}^{1} \mathrm{e}^{2}\right)\right]=0 .
$$

Отсюда, принимая во внимание линейную независимость функций $\exp \left(i 2 \pi a m x_{1}\right)$, ортогональность $\mathbf{e}^{\alpha}$, а также учитывая, что $\sigma_{0 n}^{1}=0$, получйм 


$$
\begin{aligned}
& u^{0}=0, \\
& \sum_{|n| \leqslant N} \mathrm{Q}_{m n}^{1} u_{m n}=0, \quad \sum_{|n| \leqslant N} \boldsymbol{\sigma}_{m n}^{1} u_{m n}=0, \quad|m| \leqslant M .
\end{aligned}
$$

Эти уравнения определяют пространство $V(M, N)$ уже как подпространство $S(M, N)$. Условие (24) означает, что при первой краевой задаче несжимаемая жидкость не может перемещаться как твердое тело в направлении $\mathbf{e}^{2}$.

Из вариационного уравнения (6) получим с учетом связей (24) и (25) уравнения движения

$$
\dot{u}_{m n}+F_{m n}\left(\mathbf{u}+\mathbf{v}_{0}\right)-f_{m n}=\alpha_{m} \stackrel{1}{m n}^{2}+\beta_{m} \sigma_{m n}^{1},
$$

где

$$
f_{m n}=\left(\mathbf{f}, \vec{\varphi}_{m n}^{1}\right), F_{m n}(\mathbf{v})=i k_{m n}^{\alpha}\left(\left[r v^{\alpha} \mathbf{v}-\mathbf{v}_{;} \alpha\right], \vec{\varphi}_{m n}^{1}\right) .
$$

Уравнения (26) совместно с (25) образуют замкнутую систему для определіения неизвестных $u_{m n}, \alpha_{m}, \beta_{m}$. Компонента $u^{0}$, как следует из $(24)$, не является динамической переменной.

Исключение множителей Лагранжа $\alpha_{m}, \beta_{m}$ из системы (25), (26) приводит к уравнениям типа Коши (при выводе этих уравнений нужно учитывать равенство $\left.\sum_{|n| \leqslant N} Q_{m n}^{1} \sigma_{m n}^{1}=0 \mathrm{Vm}\right)$ :

$$
\dot{u}_{m n}=\sum_{\left|n_{\mathrm{t}}\right| \leqslant N} P_{m n_{\mathrm{t}}}\left[f_{m n_{\mathrm{t}}}-F_{m n_{\mathrm{t}}}\left(\mathbf{u}+\mathbf{v}_{0}\right)\right],
$$

где ортопроектор $\hat{P}$, действующий из $S(M, N)$ в $V(M, N)$, имеет представление

$$
\begin{aligned}
& P_{m n n_{\mathrm{t}}}=\delta_{m n_{\mathrm{t}}}-\mathrm{\varrho} \mathrm{Mnn}_{m m n_{\mathrm{t}}} / r_{m}-\sigma_{m n}^{1} \sigma_{m n_{\mathrm{t}}}^{1} / s_{m}, \\
& r_{m}=\sum_{|n| \leqslant N}\left(\varrho_{m n}^{1}\right)^{2}, \quad s_{m}=\sum_{|n| \leqslant N}\left(\sigma_{m n}^{1}\right)^{2} .
\end{aligned}
$$

При $m=0$ последнее слагаемое в выражении $P_{m n n_{1}}$ пропадает, поскольку $\sigma_{0 n}^{1}=0$. Для второй краевой задачи имеем в $S(M, N)$ следующую систему уравнений:

$$
\begin{gathered}
\dot{u}_{m n}+F_{m n}\left(\mathbf{u}+\mathbf{v}_{0}\right)-f_{m n}=\left(\alpha_{m}+\gamma_{n}\right) \mathrm{Q}_{m n}^{1}+\left(\beta_{m}+\delta_{n}\right) \sigma_{m n}^{1}, \\
u_{00}=u^{0}=0, \\
\sum_{|n|<N} \sum_{m n}^{1} u_{m n}=0, \quad \sum_{|n|<N} \sigma_{m n}^{1} u_{m n}=0, \quad|m| \leqslant M, \\
\sum_{|m|<M} \mathrm{Q}_{m n}^{1} u_{m n}=0, \quad \sum_{|m|<M} \sigma_{m n}^{1} u_{m n}=0, \quad|n| \leqslant N .
\end{gathered}
$$

Исключение множителей Лагранжа $\alpha_{m}, \beta_{m}, \gamma_{n}, \delta_{n}$ из этой системы задача не столь простая, как в случае системы $(25)-(26)$.

\section{4. Динамика в пространстве $V(M, N)$}

При получении (27) осуществляется ортогональное проектирование силы, действующей на динамическую систему в $S(M, N)$, в подпространство $V(M, N)$. K этому результату можно прийти и иным путем: построением базиса в $V(M, N)$ и проектированием силы в этот базис. 
Для построения базиса в виртуальном пространстве $V(M, N)$ нужно найти независимые решения системы (25) (для первой краевой задачи) или (30) (для второй) и ортонормировать их в метрике пространства $S(M, N)$.

Обозначим ортонормальный базис в $V(M, N)$ через $\left\{\vec{\chi}^{i}\right\}_{i=1}^{I}$, где $I=\operatorname{dim} V(M, N)$, а $\overrightarrow{\chi^{i}}=\left\{\chi_{m n}^{i}\right\}_{m=-M, n=-N}^{M} . \quad$ В координатном представлении базисные векторы имеют вид

$$
\overrightarrow{\chi^{i}}(\mathbf{x})=\sum_{|m| \leqslant M} \sum_{|n| \leqslant N} \chi_{m n}^{i} \vec{\varphi}_{m n}^{1}(\mathbf{x}) .
$$

Эти векторы являются соленоидальными, бесконечно дифференцируемыми, удовлетворяющими однородным краевым условиям

$$
\begin{array}{ll}
\left.\overrightarrow{\chi^{i}}(\mathbf{x})\right|_{x_{2}=0 ; 1}=0 & \text { (первая краевая задача), } \\
\left.\overrightarrow{\chi^{i}}(\mathbf{x})\right|_{\Gamma}=0 & \text { (вторая краевая задача) }
\end{array}
$$

и условию ортонормальности $\left(\overrightarrow{\chi^{i}}, \overrightarrow{\chi^{j}}\right)^{\prime}=\delta^{i j}$.

Представляя u и w в базисе (31), получим из вариациӧнного уравнения систему.

$$
\begin{aligned}
& \dot{u}_{i}=F_{i}\left(\mathbf{u}+\mathbf{v}_{0}\right)+f_{i}, \quad i=1, \ldots, I, \\
& \mathbf{u}=\sum_{i=1}^{I} u_{i} \overrightarrow{\chi^{i}}, \quad f_{i}=\left(\mathbf{f}, \vec{\chi}^{i}\right), \\
& F_{i}(\mathbf{v})=\left(\left[r v^{\alpha} \mathbf{v}-\mathbf{v}, \alpha\right], \vec{\chi}_{, \alpha}^{i}\right),
\end{aligned}
$$

вид которой одинаков для обеих краевых задач (но базисные функции $\overrightarrow{\chi^{i}}$, конечно, различны). Замечательное свойство этой системы - она является системой типа Коши.

\section{5. Переход к бесконечной системе}

Нами были получены различные по форме уравнения. Тем не менее при фиксированных $M, N$ и заданных краевых условиях они эквивалентны - возможен переход от одной системы к другой, а решения этих систем совпадают (при одинаковых начальных условиях). Эквивалентность имеет место при любых конечных $M, N$, что позволяет в случае предельного перехода $M, N \rightarrow \infty$ ограничиться рассмотрением только одной из этих систем. В качестве таковой выберем уравнения (32).

Если $M, N$ неограниченно возрастают, то базисные функции $\overrightarrow{\chi^{i}}$ переходят в счетную систему $\left\{\chi^{i}\right\}_{i=1}^{\infty}$ линейно независимых, аналитических в $\bar{\Omega}$ функций. При этом $\overrightarrow{\chi^{i}}$ принадлежат пространству $V$, так как они удовлетворяют однородным краевым условиям и являются бесконечно дифференцируемыми соленоидальными функциями. Следовательно, линейная оболочка системы $\left\{\hat{\chi}^{i}\right\}_{i=1}^{\infty}$ в пространстве $H^{1}$ образует подпространство в $V$. Оказывается (доказательство этого результата будет опубликовано отдельно), что в действительности имеет место равенство 


$$
\left[\left\{\hat{\chi}^{i}\right\}_{i=1}^{\infty}\right]_{H^{1}}=V \text {. }
$$

Другими словами, система функций $\left\{\chi^{i}\right\}_{i=1}^{\infty}$ фундаментальна в пространстве $V$. Из этого факта следуют два утверждения:

1. В пределе полученные нами уравнения эквивалентны вариационному уравнению (6).

2. Если решение вариационной задачи принадлежит пространству $H^{1}$, то решения конечномерных уравнений в пространствах $L(M, N)$, $S(M, N)$ или $V(M, N)$ являются приближениями этого точного решения (для второй краевой задачи сходимость решения конечномерной системы (32) к решению вариационного уравнения (6) в предположении, что $\left\{\chi^{i}\right\}_{i=1}^{\infty}$ фундаментальна в $V$, установлена в $\left[{ }^{4-6}\right]$; сходимость для первой краевой задачи доказывается аналогично).

Из равенства (33) следует также, что построение базисов в последовательности пространств $\ldots V(M, N) \subset V(M+1, N+1) \subset \ldots$ дает фундаментальную систему в $V$. Построение таких систем составит предмет отдельного исследования, здесь же мы обращаем внимание на принципиальную сторону вопроса: на возможность построения фундаментальной системы в пространстве $V$ путем погружения его в более об-

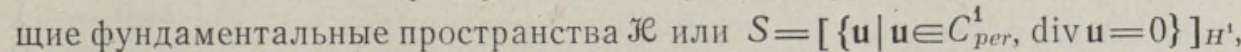
фундаментальные системы функций которых известны.

В заключение отметим, что полученные результаты можно легко обобщить на случай трехмерной прямоугольной области.

\section{Л И Т Е Р А Т У Р А}

1. Лагранж Ж. Аналитическая механика, т. 2. М.-Л., Гостехиздат, 1950.

2. Серрин Дж. Математические основы классической механики жидкости. М., 1963.

3. Парс Л. А. Аналитическая динамика. М., «Наука», 1971.

4. Ладыженская O. А. Математические вопросы динамики вязкой несжимаемой жидкости. М., «Наука», 1970.

5. Лионс Ж.-Л. Некоторые методы решения нелинейных краевых задач. М., «Мир», 1972.

6. Темам Р. Уравнения Навье-Стокса. Теория и численный анализ. М., «Мир», 1981.

7. Лионс Ж.-Л., Мадженес Э. Неоднородные граничные задачи и их приложения. М., «Мир», 1971.

8. Ахиезер А. Н., Берестецкий В. Б. Квантовая электродинамика. М., «Наука», 1969.

9. Balescu, R., Senatorski, A. Ann. Phys., 58, 587-624 (1970).
Институт астрофизики и физики атмосферы Академии наук Эстонской ССР
Поступила в редакцию 7 июня 1982

\section{R. ROOM}

\section{ÄARETINGIMUSI ARVESTAV VISKOOSSE KOKKUSURUMATU VEDELIKU DUNAAMIKA HILBERTI RUUMIS}

Virtuaalsete nihete printsiibil on kahemõõtmelises ristkülikukujulises alas voolava vedeliku liikumine projekteeritud eksponentsiaalsele Fourier' baasile. Aäretingimustel on sel juhul kitsendavate seoste iseloom ja nad põhjustavad lisaliikmete (reaktsioonijõudude) ilmumise liikumisvõrrandeisse. Töö pōhiosas on vaadeldud vōrrandite tuletamist eeldusel, et süsteemil on lõplik arv vabadusastmeid. On saadud liikumisvõrrandite erikujud solenoidaalsete vektorite ja virtuaalsete kiiruste alamruumides. Saadud võrrandid lähevad süsteemi vabadusastmete arvu piiramatult kasvades üle täpseteks NavierStokes'i võrranditeks. 


\section{DYNAMICS OF A VISCOUS INCOMPRESSIBLE FLUID, WITH BOUNDARY CONDITIONS, IN A HILBERT SPACE}

We consider the projection of the equations of motion (2) of a liquid in the rectangular region (1) to the Fourier basis (13) satisfying the incompressibility condition (3) and the boundary conditions (4) or (5). To simplify the problem, we suppose that the velocity vector $\mathbf{u}$ (see (10)) is finite-dimensional and it can be written in the form (14). Incompressibility and the boundary conditions can be treated as ideal constraints (15), (16) $((19)$ in the case of the boundary conditions (5)), giving rise to the reaction forces in the equations of motion (18), (20).

Similar equations (26), (29) have been obtained for the case of solenoidal vectors (23). These differ from the equations obtained above by absence of pressure.

Boundary conditions determine a subspace $V(M, N)$ in the space of solenoidal vectors, and the equations of motion projected to this subspace have the form (32). These equations do not contain reaction forces and belong, therefore, to the Cauchy type. Finally (section 5) we demonstrate that in the limit of infinite number of degrees of freedom the finite-dimensional model equations transform to the exact Navier-Stokes' equations for the space (33). 